\title{
Note on the text
}

For extracts from oral history transcripts used in the following text: a dash (-) indicates a hesitation; an ellipsis ( ... ) denotes a section of the transcript that has been omitted because it did not add to the quotation. Where transcripts are marked with ${ }^{\star}$ in the notes this indicates that a pseudonym has been used as the interviewee has requested anonymity. 